\title{
Bbs5 functions as the docking compartment of Bbsome binding to Klc3 regulated by Bbs5-Ser 246 PKC-phosphorylation during mouse spermatogenesis
}

Dahai $\mathrm{Yu}^{1}$, Lixia $\mathrm{He}^{1}$, Xin $\mathrm{Zhou}^{2}$, Xiuxia Wang ${ }^{1 *}$, Bingzhi $\mathrm{Yu}^{2 *}$

1. Human Reproductive Center, Shengjing Hospital of China Medical University

2. Dept. of Biochemistry, China Medical University

*Corresponding authors:

Bing-Zhi Yu

Email: Ybzbiochem@yeah.net

And

Xiu-xia Wang

Email: ydh792014@aliyun.com

Running title: PKC regulates BBS5 binding to KLC3 in mouse spermatogenesis

Key words: Protein kinase C (PKC), BBsome, BBS5, KLC3, In Vitro Phosphorylation, Spermatogenesis 


\begin{abstract}
A mitochondrial and a fibrous sheath form the midpiece of the mammalian sperm flagellum encircling most of the axoneme. It has been documented that Kinesin light chain 3 (KLC3) was involved although the formation procedure remains unclear. Yeast-two-hybrid dataset showed an interaction between Klc3 and Bardet-Biedl Syndrome 5 (BBS5) Protein, another molecular associated with cilia and flagella forming. In this study, we presumed that the most conserved IFT complex BBsome was involved in spermatogenesis via the interaction of one of its subunits, Bbs5 with Klc3. Firstly, the interaction between Klc3 and Bbs5 was confirmed with Co-IP. Secondly, we identified PKC phosphorylation sites in vitro by LC-MS/MS, Ser $^{19}$ and $\mathrm{Ser}^{246}$ of Bbs5, examined the phosphorylation status of Bbs5 Ser ${ }^{19}$ and $\mathrm{Ser}^{246}$ in mouse testis. Co-IP was performed to find which PKC isoforms phosphorylate Bbs5. In addition, we tried to discuss the roles of $\operatorname{Ser}^{19}$ and $\operatorname{Ser}^{246}$ of Bbs5 in the Klc3-bbs5 interaction and in mouse spermatogenesis based on our early findings.
\end{abstract}

\title{
Introduction
}

The eukaryotic flagellum, a highly specified organelle, is responsible for the propulsion of the male gamete in most animals. A mitochondrial and a fibrous sheath form the midpiece of the sperm flagellum encircling most of the axoneme. Kinesins are a family of proteins, essential for the proper function of many polar cells, including epithelial cells, neurons, and sperm. Spermatogenesis is closely associated with many different kinesins [1]. Kinesin light chain 3 (KLC3) is the only known kinesin light chain expressed in post-meiotic male gamete [2]. Data show that spermatids KLC3 binds to outer dense fibers and mitochondrial sheath of midpiece of sperm tail [2, 3], and low expression of KLC3 might be associated with oligozoospermia and asthenozoospermia [4]. But a lot of details remain unknown.

The BBSome, consisting of BBS1, BBS2, BBS4, BBS5, BBS7, BBS8, BBS9, and BBS18 (also known as BBIP1) is a highly conserved protein complex regulating IFT in cilia and flagella, and mutations of BBSome subunits cause Bardet-Biedl Syndrome (BBS) [5-14]. In our previous study, BBS5 assembles into the BBsome as a peripheral, non-framework subunit [10]. Novel BBs5 mutations have been eventually found but its actual function remains very limited understood. Recent studies indicated that, in retina, Bbs5/Bbs5L (a newly found splice variant) localize at axoneme via binding to arrestin1 in a phosphorylation-dependent manner, and BBs5/Bbs5L are phosphorylated by protein kinase C (PKC) $[15,16]$. The Bbs5 structural properties in BBsome and the Bbs5-arrestin1 binding indicated that Bbs5 might be functioning as a docking compartment of BBsome, and the covalent modifications of phosphorylation might be a good entry point to investigate the BBS5 functions. Meanwhile, no data has been show about the PKC phosphorylation sites of Bbs5. As a pivotal role in various biological processes, PKC also takes part in 
spermatogenesis [17-19]. Bbs5 might be one target how PKC get involved in since BBS5 is the subunit of BBsome, an indispensable complex during ciliogenesis and spermatogenesis $[20,21]$. We predicted Bbs5 as a potential PKC substrate in mice with the DISPHOS software [22], and two potential PKC phosphorylation sites, including Ser $^{19}$ and Ser $^{246}$, were predicted.

A systematic proteome-scale mappping of $\sim 14,000$ binary protein-protein interactions has inspired us in further functional investigation on both Klc3 and Bbs5. Yeast-two-hybrid dataset indicated an interaction between Klc3 and Bbs5 [23] although a confirmation is still needed. Are this interaction co-related with spermatogenesis? If yes, what is regulating this interaction? Would the 2 "predicted" phosphorylation sites take some kind of roles in it? This study was trying to address these presumptions. Initially, we began with the Co-IP to confirm the Klc3-Bbs5 interaction as a supplement of yeast-two-hybrid result [23]. Then, for the first time, by LC-MS/MS analysis following in vitro phosphorylation, we identified the PKC phosphorylation sites including $\operatorname{Ser}^{19}$ and $\operatorname{Ser}^{246}$ of Bbs5, consistent with the DISPHOS prediction. Additionally We found the various phosphorylation statuses of $\operatorname{Ser}^{19}$ and $\operatorname{Ser}^{246}$ of Bbs5 in mouse brain and testis with customized phospho/nonphospho-Bbs5(Ser19) and phospho/nonphospho-Bbs5(Ser246) antibodies, showing that $\operatorname{Ser}^{19}$ is phosphorylated in both brain and testis, while $\operatorname{Ser}^{246}$ is only phosphorylated in brain, not in testis. Then we proved that Bbs5 is the direct downstream substrate of several dominant PKC isoforms in mouse testis. At this point, we seemingly have some confidence that $\operatorname{Ser}^{19}$ and $\operatorname{Ser}^{246}$ are certain targets of PKC, and potentially regulate of Klc3-Bbs 5 interaction. The phosphorylation/dephosphorylation of Bbs5 $\operatorname{Ser}^{19}$ and $\operatorname{Ser}^{246}$ were mimicked. Consistent with the expression data, different combinations of mimicking phospho/dephospho mutants show various effects on Klc3-Bbs5 interaction, indicating that $\operatorname{Ser}^{246}$ phosphorylation/dephosphorylation may be the testis-specific switch regulating Klc3-Bbs5 interaction. Then as a supplement, more factors were introduced in: several constructs with known pathogenic mutations were built up; $\mathrm{PKC}$ was activated/deactivated by stimulator/inhibitor in media with or without serum, to observe the effects on Klc3-Bbs5 interaction.

\section{FIGURE 1.}

Co-immunoprecipitates of Klc3 with Bbs5, and Bbs4 with Bbs5. $A$, Immunoblot (IB) analysis of input cell lysates, anti-Flag immunoprecipitates (IP) derived from NIH3T3 cells transfected with pcDNA3-Flag-Kcl3 and pcDNA3-HA-Bbs5 constructs. $B$, Immunoblot (IB) analysis of input cell lysates, anti-Flag immunoprecipitates (IP) derived from NIH3T3 cells transfected with pcDNA3-Flag-Bbs4 and pcDNA3-HA-Bbs5 constructs. 


\section{Ser $^{19}$ and Ser ${ }^{246}$ Sites of Bbs5 Protein Are Phosphorylated by PKCs in Vitro}

The results of LC-MS/MS analysis of phosphorylated Bbs5 were shown in Fig. 2, $A$. Two PKC phosphorylation sites, $\operatorname{Ser}^{19}$ and $\operatorname{Ser}^{246}$, were identified.

Bardet-Biedl syndrome 5 protein homolog OS=Mus musculus $\mathrm{GN}=\mathrm{Bbs} 5 \mathrm{PE}=4 \mathrm{SV}=1$ FDVSSQQMK, Phospho(S)@5

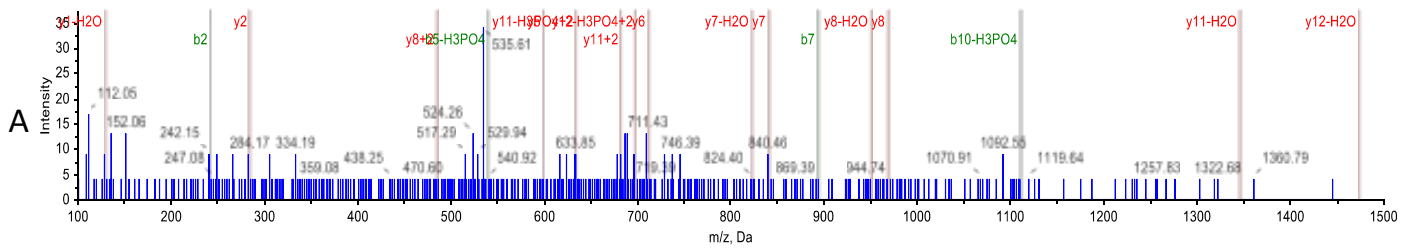

LQESVKEINSLHK, Phospho(S)@4

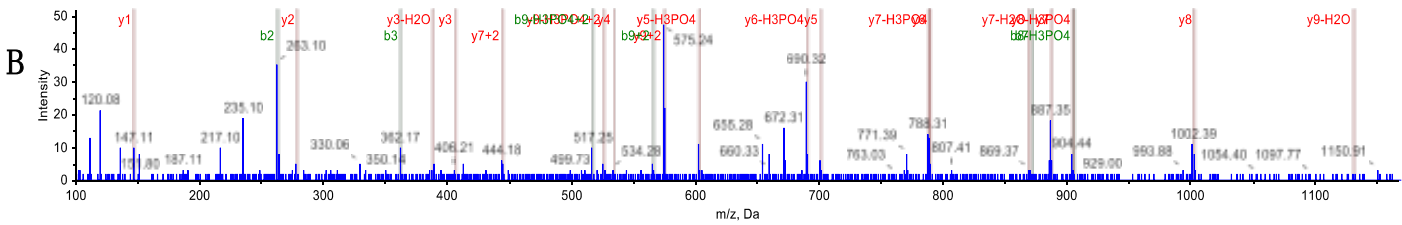

$>$ NP_082560 length $=341$

MSVLDVLWEDRDVRFDVSSQOMKTRPGEVLIDCLDSIEDTKGNNGDRGRLLVTNLRI IWHSLALPRVNL SIGYNCILNITTRTANSKLRGQTEALYILTKCNTTRFEF IFTNLVPGSPRLFTSVIAVHRAYETSKMYR

C DFKLRSAVIQNKQLRLLPQEHVYDKINGVWNLSSDQGNLGTFFITNVRIVWHANMNDSFNVSIPYLQIR SIKIRDSKFGLALVIESSQQSGGYVLGFKIDPVEKLQESVKEINSLHKVYSASPIFGVNYEMEEKPQPL EALTVEQIQDDVEIDSDDHTDAFVAYFADGNKQQDREPVFSEELGLAIEKLKDGFTLQGLWEVMS

\section{FIGURE 2.}

Bbs5 LC-MS/MS analysis identified two PKC phosphorylation sites, including Ser $^{19}$ and Ser $^{246}$.

$A$, MS spectrum of the phosphorylated peptide, ${ }^{15} \mathrm{FDVSS}^{*} \mathrm{QQMKK}{ }^{23}$, containing $\operatorname{Ser}^{19}$; $\mathrm{S}^{*}$ indicates that serine residue 19 is phosphorylated by PKC.

$B$, MS spectrum of the phosphorylated peptide, ${ }^{242}$ LQES\#VKEINSLHK ${ }^{275}$, containing $\mathrm{Ser}^{246}$, S\# indicates neutral loss of $\mathrm{H}_{3} \mathrm{PO}_{4}$ from sequence ions.

$C$, amino acid sequence of the Bbs5 protein. The $\mathrm{LC}-\mathrm{MS} / \mathrm{MS}$ analysis coverage rate of the given sample is $>97 \%$ (the covered sequence is shown in boldface letters).

The color-highlighted fragments indicate the identified phosphorylated peptide; ${ }_{p} S$ represents phosphorylated serine, including $\operatorname{Ser}(\mathrm{P})^{19}$ and $\operatorname{Ser}(\mathrm{P})^{246}$. 
Bbs5 is the direct downstream substrate of various PKC isoforms in mouse testis, Bbs5 $\operatorname{Ser}^{19}$ and Ser ${ }^{246}$ are both phosphorylated in brain, while only Se196 is phosphorylated in testis.

To identify whether Bbs5-Ser ${ }^{19}$ was phosphorylated in vivo, we collected mouse brain and testis tissue then measured the phosphorylation status of Bbs5-Ser ${ }^{19}$ and $\operatorname{Ser}^{246}$ by the phosphospecific antibodies, and the nonphosphorylation status by the nonphosphospecific antibodies. Fig. $3 A$ showed that phosphorylated Bbs5-Ser ${ }^{19}$ band was observed in both brain and testis, whereas stronger phosphorylation of Bbs5-Ser ${ }^{19}$ was observed. Interestingly, a phosphorylated Bbs5-Ser ${ }^{246}$ band was detected in brain but absent in testis. Taken together, these results demonstrated that both Bbs5-Ser ${ }^{19}$ and Bbs5-Ser ${ }^{246}$ are phosphorylated in brain while only Bbs5-Ser ${ }^{19}$ in testis, indicating a tissue specificity of Bbs5 phophorylation. Also, we are interested in which PKC isoforms interact with Bbs5 in testis and the interactions were confirmed between 5 dominant PKC isoforms in testis, PKC $\alpha, \beta, \gamma, \delta, \zeta$ and Bbs5.

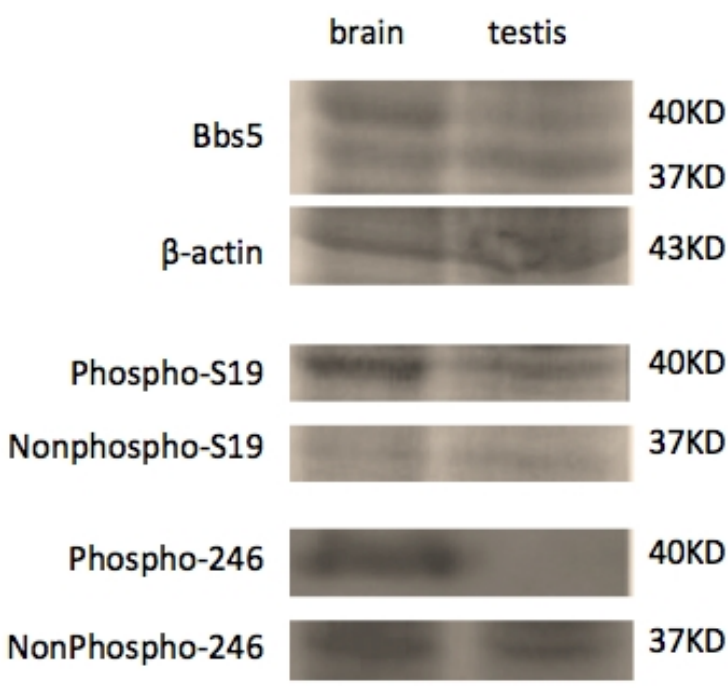

A
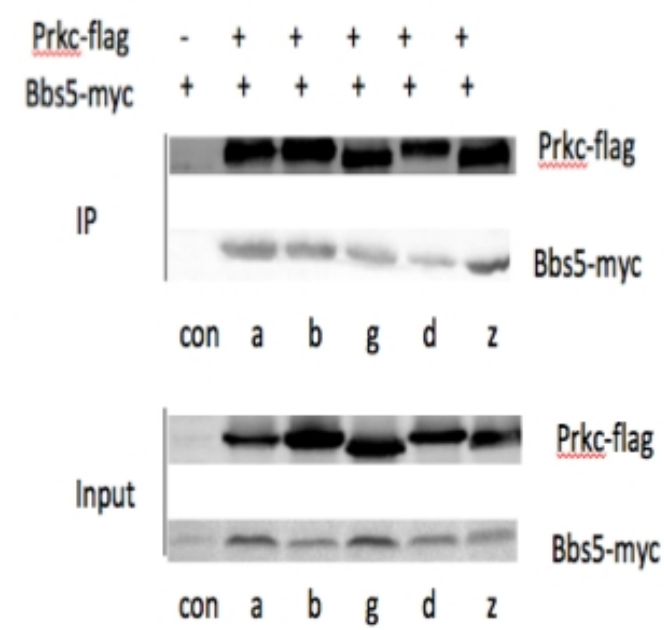

\section{FIGURE 3.}

Endogenous Bbs5 protein expression and detection of the phosphorylation status of Bbs5-Ser ${ }^{19}$ and Bbs5-Ser ${ }^{246}$ in mouse brain and testis by Western blotting. $A$, phosphorylated Bbs5-Ser ${ }^{19}$ band was observed in both testis and $\mathrm{S}$ brain, whereas stronger phosphorylation of Bbs5-Ser ${ }^{19}$ was observed; a phosphorylated Bbs5-Ser ${ }^{246}$ band was detected in brain other than in testis. The molecular mass of proteins is indicated on the right side of the figure. $\boldsymbol{B}$, Immunoblot (IB) analysis of input cell lysates, anti-Flag immunoprecipitates (IP) derived from NIH3T3 cells transfected with Prkc-flag and Bbs5-myc constructs. 


\section{Simulated phosphorylation/nonphosphorylation on single Ser site or both Ser sites regulate the Bbs5-Klc3 interaction.}

6 site-directed mutantgenesis Bbs5 constructs were built up, with Simulated phosphorylation/nonphosphorylation mutations on $\mathrm{Ser}^{19}$, $\mathrm{Ser}^{246}$ or both $\mathrm{Ser}^{19} / \mathrm{Ser}^{246}$. Simulated phosphorylation/nonphosphorylation on Ser ${ }^{19}$ have no effect on Bbs5-Klc3 interaction (Fig. 4 A.), indicating this interaction an intrinsic property of Bbs5 binding to Klc3; Interestingly, simulated phosphorylation on $\mathrm{Ser}^{246}$ completely block Bbs5-Klc3 interaction regardless of $\operatorname{Ser}^{19}$ (Fig. 4 B, C.), consistent with the Endogenous Bbs5 expression data.
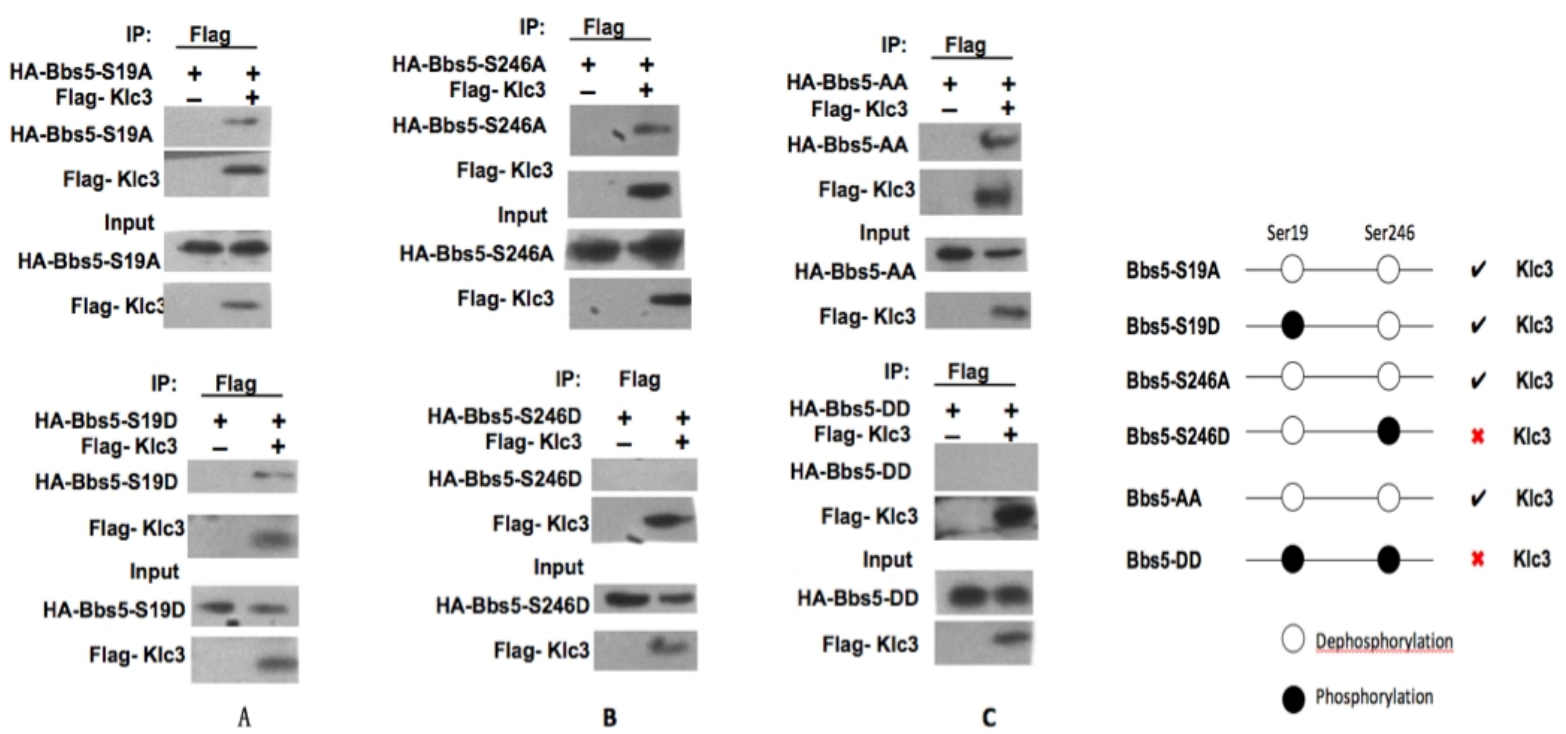

FIGURE 4.

Co-IP of Klc3 with Bbs5 mutants, Bbs5-S19A, Bbs5-S246A, Bbs5-S19A/S246A, Bbs5-S19D, Bbs5-S246D, or Bbs5-S19D/S246D.

$A$, Immunoblot (IB) analysis of input cell lysates, anti-Flag immunoprecipitates (IP) derived from NIH3T3 cells transfected with pcDNA3-Flag-Kcl3 and pcDNA3-HA-Bbs5 S19A/S19D constructs. $B$, Immunoblot (IB) analysis of input cell lysates, anti-Flag immunoprecipitates (IP) derived from NIH3T3 cells transfected with pcDNA3-Flag-Klc3 and pcDNA3-HA-Bbs5 S246A/S246D constructs. $C$, Immunoblot (IB) analysis of input cell lysates, anti-Flag immunoprecipitates (IP) derived from NIH3T3 cells transfected with pcDNA3-Flag-Klc3 and pcDNA3-HA-Bbs5 S19A+S246A/S19D+S246D constructs.

Some confirmed pathogenic Bbs5 mutations do not interrupt the Klc3-Bbs5 interaction. 
Additionally, We tried to observe whether several known BBs5 mutations could affect this interaction [23-25]. Only the pathogenic missense mutants were picked up for making the corresponding constructs. Co-IPs were performed and the data shows that none of mutations could $100 \%$ block the Klc3-Bbs5 interaction, while only G72S show a weakened pulled-down. More attention would be paid on in future study.

Table 1. Mutations Reported in BBS5

\begin{tabular}{|c|c|c|}
\hline c.DNA & Predicted effect & Mutation type \\
\hline c.166A $>$ G & p.R56G & Pathogen/ missense \\
\hline c.149T $>$ G & p. Leu50R & Pathogen/ missense \\
\hline c.123delA & p.Gly42GlnfsX11 & Nonsense \\
\hline 263_271indelGCTCTTA & Indel-1 fs X & Nonsense \\
\hline c. $176 \mathrm{G}>\mathrm{A}$ & p.Trp59X & Nonsense \\
\hline c.214G $>$ A & p.Gly72Ser & Pathogen/ missense \\
\hline c. $181 \mathrm{~T}>\mathrm{A} / \mathrm{G}$ & p.Leu142X & Nonsense \\
\hline IVS6+3A $>\mathrm{G} 1$ & fsX in exon 7 & Nonsense \\
\hline c.547G $>$ A & p.Thr183Ala & Pathogen/ missense \\
\hline c.551A $>\mathrm{G}$ & p.Asn184Ser & Conflicting interpretations of \\
& & pathogenicity \\
\hline
\end{tabular}
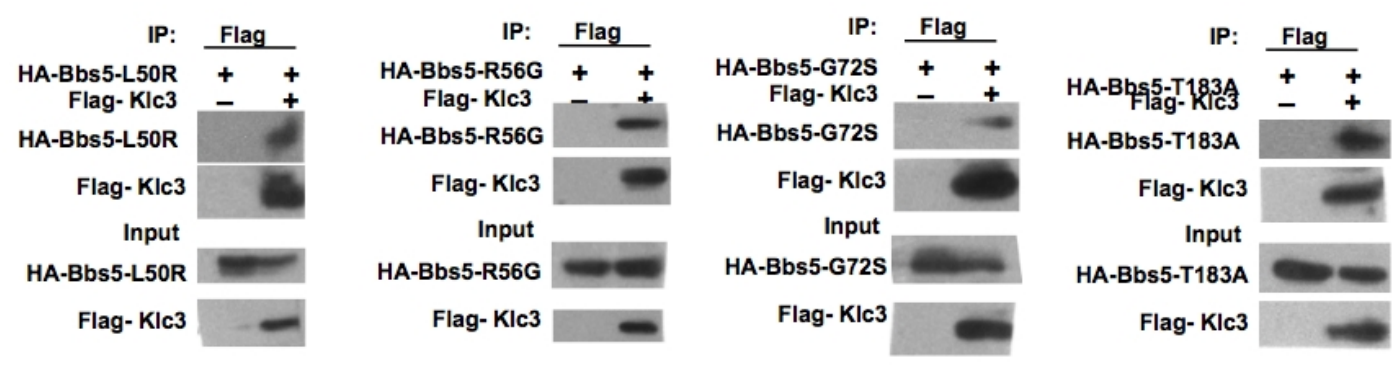

FIGURE 5.

Co-IP of Klc3 with Bbs5 mutants, Bbs5-L50R, Bbs5-R56G, Bbs5-G72S and Bbs5-T183A.

Immunoblot (IB) analysis of input cell lysates, anti-Flag immunoprecipitates (IP) derived from NIH3T3 cells transfected with pcDNA3-Flag-Klc3 and pcDNA3-HA-Bbs5 mutants constructs. 
All previous results come to that the Klc3-Bbs5 binding in testis is more acting like the intrinsic assembly under the various phosphorylation status combination of Ser19 and Ser246. We also want to understand what if the PKC was just stimulated or inhibited. The Klc3-Bbs5 binding was observed again under several different treatments. In the medium with/without serum, the PKC stimulator TPA or inhibitor H7 was applied to up-regulate or down-regulate the PKC activity. Apparently, serum starvation itself does not block the binding. Surprisingly, both the stimulation and the inhibition of PKC would almost $100 \%$ block the interaction.

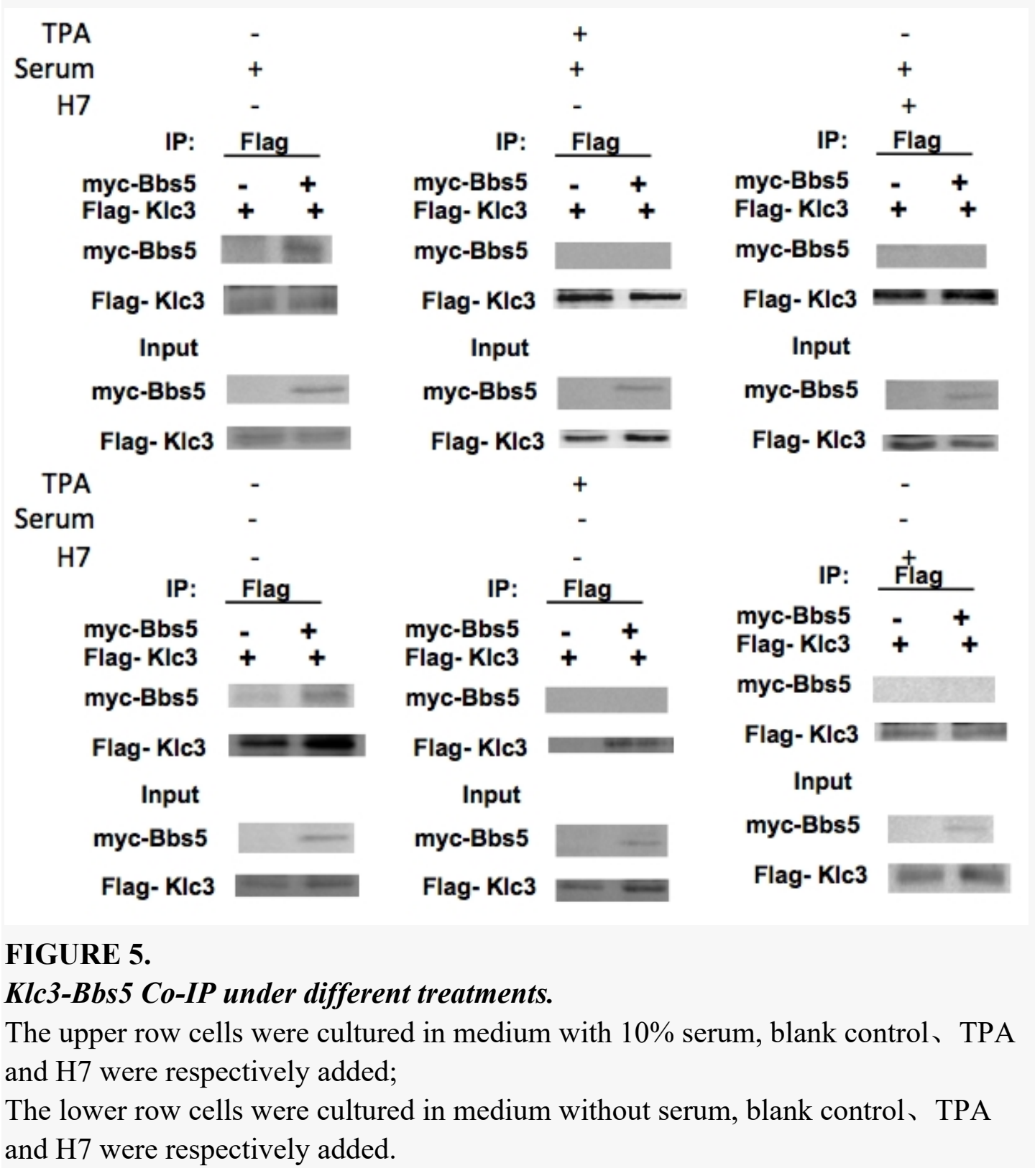




\section{DISCUSSION}

Eukaryotic flagella and cilia share a basic 9+2 microtubule-organization of axonemes, and could generate bending-motion [28]. The eukaryotic flagellum, generally considered as a highly specified cilium, is responsible for the propulsion of the male gamete in most animals. Compatible with this function, a mitochondrial and a fibrous sheath has been evolved to form the midpiece of the sperm flagellum, encircling most of the axonemes. Kinesins are a family of proteins, essential for the proper function of many polar cells, including epithelial cells, neurons, and sperms. Spermatogenesis is closely associated with many different kinesins [1]. Kinesin light chain 3 (KLC3), a member of the kinesin-1 family, is the only known kinesin light chain expressed in postmeiotic male gamete [2]. It contains a conserved heptad repeat (HR) binding to kinesin heavy chain (KHC) [29, 30] and tandem tetratrico-peptide repeats (TPR) mediating protein interactions in [31]. KLC3 are highly expressed in mouse round and elongating spermatids. KLC3 accumulates in the sperm tail midpiece consisting of a mitochondrial sheath [32]. KLC3 binds to mitochondria during rat spermatogenesis and was believed functioning either as a vehicle or a fixation [33]. Low expression of KLC3 might be associat6hged with oligozoospermia and asthenozoospermia [4]. KLC3 is proposed to play multiple roles in sperm tail development. But a lot of details remain unknown.

BBS5 is one of the known 20 pathogenic genes causing Bardet-Biedl Syndrome (BBS) [5-14]. The BBSome, consisting of BBS1, BBS2, BBS4, BBS5, BBS7, BBS8, BBS9, and BBS18 (also known as BBIP1) is a highly conserved protein complex regulating IFT in cilia and flagella. In my sequential assembly model, BBS5 assembles into the BBsome as a peripheral, non-framework subunit [10]. Novel BBs5 mutations are still being found eventually [23-25], but its actual function remains very limited understood. The two proteins appear to have been separated and independent, but the biological function they are both involved in show some potential coincidence. A systematic proteome-scale mappping has investigated $\sim 14,000$ binary protein-protein interactions by yeast-two-hybrid system. The huge dataset indicated an interaction between Klc3 and Bbs5 [23], which inspired us in further functional study on the relation of Klc3 and Bbs5. First of all, the protein-protein interaction was confirmed with Co-Immunopercipitation. Flag-tagged Klc3 and HA-tagged Bbs5 could pulled down each other (see Fig. 1), which means we already set a link between the Klc3 and BBsome. And we presumed that Klc3 might be functioning as a vehicle with the binding of the IFT Cargo complex, BBsome.

Recent studies indicated that, in retina, Bbs5/Bbs5L (a newly found splice variant) localize at axonemes via binding to arrestin1 in a phosphorylation-dependent manner, and BBs5/Bbs5L are phosphorylated by protein kinase $\mathrm{C}$ (PKC) $[15,16]$. The Bbs5 assembling properties in BBsome and the Bbs5-arrestin1 binding indicated that Bbs5 might be functioning as a docking compartment of BBsome, and the covalent 
modifications of phosphorylation might be a good entry point to investigate the BBS5 functions. For the time being, there is still no data about the PKC phosphorylation sites of Bbs5. As an important role in various biological processes, $\mathrm{PKC}$ also takes part in spermatogenesis [17-19]. Bbs5 might be one potential target of which PKC gets involved in since BBS5 is the subunit of BBsome, an indispensable complex during ciliogenesis and spermatogenesis $[20,21]$. We analyzed Bbs5 as a potential PKC substrate in mice with the DISPHOS software [22], and two PKC phosphorylation sites, including $\operatorname{Ser}^{19}$ and $\mathrm{Ser}^{246}$, were predicted. But no data has been published about the exact phosphorylation sites. We performed the in vitro phosphorylation with purified Bbs5 protein as the substrate of $\mathrm{PKC}$, and then the sample was analyzed with LC-MS/MS. Both $\operatorname{Ser}^{19}$ and Ser $^{246}$ site were confirmed, consistent with DISPHOS prediction (see Fig. 2). The next question we need to figure out would be these sites function. We detected the endogenous Bbs5 expression in mouse brain and testis, with the customized Bbs5 phospho/nonphospho antibodies recognizing either site. Stronger phosphorylation of Bbs5-Ser ${ }^{19}$ was observed in both brain and testis. Interestingly, the phosphorylated Bbs5-Ser ${ }^{246}$ band was detected in brain but absent in testis (see Fig. 3. A). These results demonstrated that both Bbs5-Ser ${ }^{19}$ and Bbs5-Ser ${ }^{246}$ are phosphorylated in brain while only Bbs5-Ser ${ }^{19}$ in testis, suggesting that Bbs5 is phosphorylated in a tissue-specific pattern. And the dephosphorylation of Bbs5-Ser ${ }^{246}$ might play some role in Bbs5's testis specific function. Also, we were interested in whether any PKC isoforms specifically phosphorylate Bbs5 in testis. The interactions were confirmed with Co-Ips, between 5 dominant PKC isoforms in testis, PKC $\alpha, \beta, \gamma, \delta, \zeta$ and Bbs5 while no significant difference was observed (see Fig. 3. B).

We further explored the role of Bbs5-Ser ${ }^{19}$ and $\mathrm{Bbs} 5-\mathrm{Ser}^{246}$ in regulating the Klc3-Bbs5 interaction. HA-Tagged De-phosphomimic and phosphomimic mutants constructs, Bbs5-S19A/S19D, Bbs5-S246A/S246D and Bbs5-S19A-S246A/S19D-S246D, were respectively co-overexpressed with Flag-tagged Klc3 at the present of PKC inhibitor $\mathrm{H} 7$ to down-regulate the endogenous phosphorylation. Surprisingly, Our findings showed functions of Bbs5-Ser ${ }^{19}$ and Bbs5-Ser ${ }^{246}$ in Klc3-Bbs5 interaction in diverse directions. Simulated phosphorylation/nonphosphorylation on $\operatorname{Ser}^{19}$ apparently have no effect on Bbs5-Klc3 interaction (see Fig. 4. A), suggesting that binding to Klc3 might be an intrinsic property of Bbs5 in mouse testis, regardless of Ser $^{19}$ phosphorylation status; Interestingly, simulated phosphorylation on $\operatorname{Ser}^{246}$ completely block Bbs5-Klc3 interaction regardless of $\operatorname{Ser}^{19}$ (see Fig. 4. B \& C), consistent with the tissue endogenous Bbs5 expression data (see Fig. 3. A).

Additionally, We tried to observe whether several known BBS5 mutations could affect this interaction [23-25]. In this study, only the pathogenic missense mutants generating a full length Bbs5 protein were picked up for making the corresponding constructs. Co-IPs were performed and the data shows that none of mutations could 
$100 \%$ block the Klc3-Bbs5 interaction, while only G72S show a weakened pulled-down. More investigation needs to be done in future.

Our current results come to that the Klc3-Bbs5 binding in testis is more acting like the intrinsic assembly under the different phosphorylation status of Bbs5-Ser ${ }^{19}$ and Ser ${ }^{246}$. We also want to understand what if the PKCs were just diffusely stimulated or inhibited. The Klc3-Bbs5 binding was observed again under several different conditions. In the medium with/without serum, the PKC stimulator TPA or inhibitor H7 was applied to up-regulate or down-regulate the PKCs activity. Apparently, serum starvation itself does not block the binding. But surprisingly, both the stimulation and the inhibition of PKCs would almost $100 \%$ block the interaction, suggesting the diffuse stimulation and inhibition of PKCs might regulate Klc3-Bbs5 interaction in some alternative mechanism not fully elucidated. A hypothesis for right now would be the phosphorylation status of Bbs5-Ser ${ }^{19}$ and $\operatorname{Ser}^{246}$ were regulated by different PKC isoforms.

Collectively, the aforementioned findings could be accommodated in the following working model. In testis, the Bbs5 in fully assembled BBsome provides a docking site for Klc3. This docking site for Klc3 is only available in condition of Bbs5-Ser246 dephosphorylated. This testis-specific BBsome-Klc3 complex functions as a transportation cargo for the mitochondria to the designated subcellular localization. The temporary Bbsome-Klc3 complex releases Klc3 at the midpiece of sperm tail. Then Klc3 will continue executing other functions. And the BBsome keeps going along the flagellum.

In the future, it is of great importance to probe the exact mechanisms modulating the association of Klc3 with Bbs5, regulation of dephosphorylation of Bbs5-Ser ${ }^{246}$ and the other functions of diverse phosphorylation of Bbs5-Ser ${ }^{19}$ and Bbs5-Ser ${ }^{246}$ in various tissues.

\section{Experimental procedures}

Kunming genealogy-specific pathogen-free mice (males at 8 weeks and $\sim 30$ g) were obtained from the Department of Laboratory Animals, China Medical University. All experiments were performed at China Medical University in accordance with the National Institutes of Health guidelines for the Care and Use of Laboratory Animals. Reagents, unless otherwise specified, were purchased from Sigma-Aldrich.

\section{Prokaryotic Expression and Purification of Recombinant Bbs5 Protein}

A 1026-bp fragment encoding 341 amino acids was amplified by PCR with mouse testis cDNAs library as template, and PCR was performed with 35 cycles (primers: 5'-CGCGGATCCATGTCTGTGCTGGACGTGTTG $-3^{\prime}$ and $5^{\prime}$-CCGCTCGAGTCAACTCATCACTTCCCAAAGTC-3'). Each PCR cycle consisted of $30 \mathrm{~s}$ at $94{ }^{\circ} \mathrm{C}, 40 \mathrm{~s}$ at $64{ }^{\circ} \mathrm{C}$, and $60 \mathrm{~s}$ at $72{ }^{\circ} \mathrm{C}$. The PCR product was then 
subcloned into the pGEX-4T-2 vector. The recombinant plasmids, pGEX-4T-2-Bbs5-WT, was sequenced to verify the correct insertion.

The GST-Bbs5 was expressed for $3 \mathrm{~h}$ at $27^{\circ} \mathrm{C}$ in Escherichia coliBL21(DE3) (Takara) transformed with pGEX-4T-2-Bbs5-WT in the presence of $0.1 \mathrm{mM}$ isopropyl- $\beta$-D-thiogalactopyranoside (Takara). The proteins were purified with the glutathione-Sepharose 4B protein chromatography purification kit (GE Healthcare). Total protein was induced by isopropyl- $\beta$-D-thiogalactopyranoside, vector-expressed protein, and purified protein was identified with 10\% SDS-PAGE and Western blotting.

\section{Phosphorylation of GST-Bbs5 Fusion Protein in Vitro and LC-MS/MS Analysis of PKC Phosphorylation Site}

Purified GST-Bbs5 protein $(400 \mu \mathrm{g})$ was incubated with $6 \mu \mathrm{l}$ of PKC (New England Biolabs) and $10 \mu \mathrm{l}$ of ATP (New England Biolabs) at $30{ }^{\circ} \mathrm{C}$ for $45 \mathrm{~min}$. Following $10 \%$ SDS-PAGE separation, the band of phosphorylated Bbs5 protein was excised from the gel, and in-gel digestion was performed. To obtain maximal sequence coverage of protein, the digested products were collected at 1, 2, 4, and $12 \mathrm{~h}$. The sample was combined and analyzed with LC-linear trap quadrupole-MS/MS (Thermo Fisher Scientific, Waltham, MA) with MS/MS (MS') and MS/MS/MS (MS 3 ) strategy. MS data were searched with SEQUEST, and the spectra of all identified phosphor-peptides were manually verified.

\section{Plasmid Construction and Site-directed Mutagenesis}

A 1053-bp fragment encoding 5'-HA-tagged Bbs5 was amplified by PCR with pGEX-4T-2-Bbs5-WT as template, and PCR was performed with 35 cycles (primers: 5'-CACGGATCCATGTACCCATACGATGTTCCAGATTACGCT TCTGTG-3' and 5'-CCGCTCGAGTCAACTCATCACTTCCCAAAGTC-3'). The PCR product was then subcloned into the pCDNA3 vector. The recombinant plasmids, pcDNA3-HA-Bbs5-WT, was sequenced to verify the correct insertion. The pcDNA3-HA-Bbs5-S19A construct was prepared by mutating Ser ${ }^{19}$ of Bbs5 to alanine with pcDNA-HA-Bbs5-WT as template and site-directed mutagenesis kit (Stratagene). The primers were FWD1 (5'CTTCGACGTGTCCGCACAACAGATGAAAAC-3') and REV2 (5'GTTTTCATCTGTTGTGCGGACACGTCGAAG-3'). The pcDNA3-HA-Bbs5-S246A or pcDNA3-HA-Bbs5-S19A/S246A constructs were prepared by mutating Ser ${ }^{246}$ of Bbs5 to alanine with pcDNA3-HA-Bbs5-WT or pcDNA3-HA-Bbs5-S19A as templates. The primers were FWD3 (5'-GTGGAAAAACTACAGGAAGCAGTTAAAGAGATCAACTCAC-3') and REV4 (5'-GTGAGTTGATCTCTTTAACTGCTTCCTGTAGTTTTTCCAC-3'). Mutants mimicking phosphorylation, pcDNA3-HA-Bbs5-S19D, pcDNA3-HA-Bbs5-S246D and pcDNA-HA-Bbs5-S19D/S246D, were also constructed, in which residues 19 and/or 246 were replaced by aspartic acid. The primers were FWD5 (5'-CTTCGACGTGTCCGACCAACAGATGAAAAC -3'), REV6 (5'-GTTTTCATCTGTTGGTCGGACACGTCGAAG-3'), FWD7 (5'- 
GTGGAAAAACTACAGGAAGACGTTAAAGAGATCAACTCAC-3') and REV8 (5'-GTGAGTTGATCTCTTTAACGTCTTCCTGTAGTTTTTCCAC-3'). With similar strategy, pcDNA3-myc-Bbs5-WT, pcDNA3-HA-Bbs5-L50R, pcDNA3-HA-Bbs5-R56G, pcDNA3-HA-Bbs5-G72S, pcDNA3-HA-Bbs5-T183A, pcDNA3-flag-Bbs4, pcDNA3-flag-Klc3 and pcDNA3-flag-PKC- $\alpha / \beta / \gamma / \delta / \zeta$ were also built up. All of the above recombinant plasmids were sequenced to verify the correct gene insertion and successful mutation.

\section{Western Blotting and Co-Immunoprecipitation}

Mouse brain/testis and transfected NIH3T3 cells were lysed, subjected to SDS-PAGE $(10 \%)$, transferred onto nitrocellulose membranes, and immunoblotted with antibodies to Bbs5 (1:200; Santa Cruz Biotechnology), phospho-Bbs5-pSer ${ }^{19}$ /nonphospho-Bbs5-Ser ${ }^{19}$, phospho-Bbs5-pSer ${ }^{246} /$ nonphospho-Bbs5-Ser ${ }^{246}$ (1:300; Bioyear Gene), GST/HA/Myc/Flag and $\beta$-actin (1:500; Beyotime Institute of Biotechnology). Proteins were visualized by the enhanced chemiluminescence (ECL) detection system (Pierce Biotechnology). The phospho-Bbs5-pSer ${ }^{19} /$ nonphospho-Bbs5-Ser $^{19}$ and phospho-Bbs5-pSer ${ }^{246} /$ nonphospho-Bbs5-Ser ${ }^{246}$ antibodies were raised in New Zealand White Rabbits against the keyhole limpet hemocyanin-conjugated phosphopeptides, DRDVRFDVSpSQQMKTRPG / DPVEKLQEpSVKEINSLHK, or the nonphosphopeptides, DRDVRFDVSSQQMKTRPG DPVEKLQESVKEINSLHK.

\section{Funding and additional information}

* This work was supported by Liaoning Province Education Administration Doctoral Startup Fund Grant 201501011.

\section{Conflict of interest}

The authors declare that they have no conflicts of interest with the contents of this article.

\section{References}

1. Ma D D, Wang D H, Yang W X. Kinesins in spermatogenesis[J]. Biology of Reproduction, 2017, 96(2):267-276.

2. Zhang Y, Oko R, Hoorn F A V D. Rat kinesin light chain 3 associates with spermatid mitochondria[J]. Developmental Biology, 2004, 275(1):23.

3. Zhang $\mathrm{Y}, \mathrm{Ou} \mathrm{Y}$, Cheng $\mathrm{M}$, et al. KLC3 is involved in sperm tail midpiece formation and sperm function[J]. Developmental Biology, 2012, 366(2):101 
4. Dastjerdy P K, Tavalaee M, Salehi M, et al. Altered expression of KLC3 may affect semen parameters[J]. International Journal of Reproductive Biomedicine, 2016, 14(1):15-22.

5. Mykytyn K, Sheffield VC. Establishing a connection between cilia and Bardet-Biedl Syndrome. Trends Mol Med. 2004;10(3):106-9.

6. Nishimura DY, Searby CC, Carmi R, Elbedour K, Van Maldergem L, Fulton AB, et al. Positional cloning of a novel gene on chromosome $16 \mathrm{q}$ causing Bardet-Biedl syndrome (BBS2). Human Molecular Genetics. 2001;10(8):865-74.

7. Mykytyn K, Braun T, Carmi R, Haider NB, Searby CC, Shastri M, et al. Identification of the gene that, when mutated, causes the human obesity syndrome BBS4. Nat Genet. 2001;28(2):188-91.

8. Nishimura DY, Swiderski RE, Searby CC, Berg EM, Ferguson AL, Hennekam R, et al. Comparative genomics and gene expression analysis identifies BBS9, a new Bardet-Biedl syndrome gene. The American Journal of Human Genetics. 2005;77(6):1021-33.

9. Nachury MV, Loktev AV, Zhang Q, Westlake CJ, Peranen J, Merdes A, et al. A core complex of BBS proteins cooperates with the GTPase Rab8 to promote ciliary membrane biogenesis. Cell. 2007;129(6):1201-13.

10. Zhang QH, Yu DH, Seo SJ, Stone EM, Sheffield VC. Intrinsic Protein-Protein Interaction-mediated and Chaperonin-assisted Sequential Assembly of Stable Bardet-Biedl Syndrome Protein Complex, the BBSome.Journal of Biological Chemistry. 2012;287(24):20625-35.

11. Scheidecker S, Etard C, Pierce NW, Geoffroy V, Schaefer E, Muller J, et al. Exome sequencing of Bardet-Biedl syndrome patient identifies a null mutation in the BBSome subunit BBIP1 (BBS18). J Med Genet. 2014;51(2):132-6.

12. Badano JL, Ansley SJ, Leitch CC, Lewis RA, Lupski JR, Katsanis N. Identification of a novel Bardet-Biedl syndrome protein, BBS7, that shares structural features with BBS1 and BBS2. Am J Hum Genet. 2003;72(3):650-8.

13. Katsanis N, Lewis RA, Stockton DW, Mai PMT, Baird L, Beales PL, et al. Delineation of the critical interval of Bardet-Biedl Syndrome 1 (BBS1) to a small region of $11 \mathrm{q} 13$, through linkage and haplotype analysis of 91 pedigrees. Am J Hum Genet. 1999;65(6):1672-9.

14. Mykytyn K, Nishimura DY, Searby CC, Shastri M, Yen HJ, Beck JS, et al. Identification of the gene (BBS1) most commonly involved in Bardet-Biedl syndrome, a complex human obesity syndrome. Nat Genet. 2002;31(4):435-8.

15. Smith T, Spitzbarth B, Li J, Dugger D, Stern-Schneider G, Sehn E, et al. (2013) Light-dependent phosphorylation of Bardet-Biedl syndrome 5 in photoreceptor cells modulates its interaction with arrestin1. Cell Mol Life Sci 70: 4603-4616.

16. Bolch S N, Dugger D R, Chong T, et al. A Splice Variant of Bardet-Biedl Syndrome 5 (BBS5) Protein that Is Selectively Expressed in Retina.[J]. Plos One, 2016, 11(2): e0148773.

17. Teijeiro J M, Marini P E, Bragado M J, et al. Protein kinase C activity in boar sperm[J]. Andrology, 2017, 5(2):381. 
18. Barón L, Fara K, Zapata - Carmona H, et al. Participation of protein kinases and phosphatases in the progesterone - induced acrosome reaction and calcium influx in human spermatozoa[J]. Andrology, 2016, 4(6):1073-1083.

19. Lackey B R, Gray S L. Identification of kinases, phosphatases, and phosphorylation sites in human and porcine spermatozoa[J]. Systems Biology in Reproductive Medicine, 2015, 61(6):1.

20. Avidorreiss T, Leroux M R. Shared and Distinct Mechanisms of Compartmentalized and Cytosolic Ciliogenesis.[J]. Current Biology Cb, 2015, 25(23):R1143.

21. Zhang Q, Nishimura D, Vogel T, et al. BBS7 is required for BBSome formation and its absence in mice results in Bardet-Biedl syndrome phenotypes and selective abnormalities in membrane protein trafficking.[J]. Journal of Cell Science, 2013, 126(11):2372-2380.

22. http://www.dabi.temple.edu/disphos/pred/predict

23. [dataset]Rolland T, Taşan M, Charloteaux B, et al. A proteome-scale map of the human interactome network[J]. Cell, 2014, 159(5):1212.

24. Muller J, Stoetzel C, Vincent M C, et al. Identification of 28 novel mutations in the Bardet-Biedl syndrome genes: the burden of private mutations in an extensively heterogeneous disease.[J]. Human Genetics, 2010, 127(5):583.

25. Hjortshøj T D, Grønskov K, Philp A R, et al. Novel mutations in BBS5 highlight the importance of this gene in non-Caucasian Bardet-Biedl syndrome patients[J]. American Journal of Medical Genetics Part A, 2008, 146A(4):517-20.

26. M'Hamdi O, Redin C, Stoetzel C, et al. Clinical and genetic characterization of Bardet-Biedl syndrome in Tunisia: defining a strategy for molecular diagnosis[J]. Clinical Genetics, 2014, 85(2):172-7.

27. Ece S A, Onay H, Atik T, et al. Targeted multi-gene panel testing for the diagnosis of Bardet Biedl syndrome: Identification of nine novel mutations across BBS1, BBS2, BBS4, BBS7, BBS9, BBS10 genes.[J]. European Journal of Medical Genetics, 2015, 58(12):689-694.

28. Pigino G, Maheshwari A, Bui K H, et al. Comparative structural analysis of eukaryotic flagella and cilia from Chlamydomonas, Tetrahymena, and sea urchins.[J]. Journal of Structural Biology, 2012, 178(2):199-206.

29. Stout J R, Rizk R S, Kline S L, et al. Deciphering protein function during mitosis in PtK cells using RNAi[J]. BMC Cell Biology, 2006, 7(1):26.

30. Rizk R S, Bohannon K P, Wetzel L A, et al. MCAK and Paclitaxel Have Differential Effects on Spindle Microtubule Organization and Dynamics[J]. Molecular Biology of the Cell, 2009, 20(6):1639.

31. Jr J G G, Goldstein L S B. Tetratrico peptide repeats are present in the kinesin light chain[J]. Trends in Biochemical Sciences, 1996, 21(2):52-53.

32. Junco A, Bhullar B, Tarnasky H A, et al. Kinesin light-chain KLC3 expression in testis is restricted to spermatids.[J]. Biology of Reproduction, 2001, 64(5):1320.

33. Zhang Y, Oko R, Fa V D H. Rat kinesin light chain 3 associates with spermatid mitochondria.[J]. Developmental Biology, 2004, 275(1):23. 